\title{
Factors Influencing Self-care Behaviors in Elderly Hemodialysis Patients
}

\author{
Su-Jeong $\mathrm{Han}^{1}$ and Hye-Won $\mathrm{Kim}^{2} *$ \\ ${ }^{1}$ Department of Nursing, Konyang University \\ ${ }^{2}$ *Seoul Women's College of Nursing \\ 'sjhan@konyang.ac.kr \\ 2,hwkim@snjc.ac.kr
}

\begin{abstract}
A growing number of patients are supported by dialysis in older age. Care of these patients can be challenging due to chronic healthcare conditions, diminished quality of life, and an increased risk of death. The purposes of this study were to measure self-care behaviors in elderly hemodialysis (HD) patients and to identify variables associated with self-care behaviors. The subjects were 113 elderly patients on HD who visited a tertiary hospital in Seoul between November $5^{\text {th }}$ and $30^{\text {th }}, 2012$, and consented to participate in the study. The SPSS WIN 15.0 program was used of data analysis. The mean score for self-care behaviors was 3.14. Caring for an arteriovenous fistula, followed by taking medication showed the highest scores for self-care activities, and engaging in social activities and diet control showed the lowest scores. The mean score for social support was 76.8. Significant negative correlation was observed between self-care behaviors and serum phosphorus, and potassium concentration, and significant positive correlation was observed between self-care behaviors and social support. Age, education level, and social support predicted value accounted for $36.8 \%$ of the variance in self-care behaviors in elderly hemodialysis patients. Dialysis providers should recognize self-care behaviors as an important nursing issue for elderly hemodialysis patients, and self-care and selfefficacy training should be provided to improve patients' confidence in performing selfcare behaviors.
\end{abstract}

Keywords: self-care behaviors, social support, elderly, hemodialysis

\section{Introduction}

End-stage renal disease (ESRD) is a chronic illness. Treatment options for the disease often involve either long-term dialysis or kidney transplantation. In particular, patients over the age of 75 have higher incidence rates of ESRD than younger patients and constitute the fastest growing segment of the ESRD population worldwide [1]. The burgeoning incidence of older dialysis patients has been demonstrated in recent data from the ERA-EDTA registry [2]. Up to one-third of elderly patients with ESRD have four or more chronic health conditions when they reach ESRD, and many are not considered candidates for kidney transplantation. Therefore, the majority of elderly patients with ESRD face the prospect of dialysis therapy for the remainder of their lives. ESRD is an irreversible loss of kidney function to the point that the kidneys fail to support life.

Hemodialysis (HD) is an expensive and time consuming procedure requiring that patients follow a strict treatment schedule and fluid and dietary restrictions and therefore involves a wide range of life style changes [3-4]. In addition, these patients encounter many physical and psychosocial stresses, including hypertension, lack of appetite, anemia, sexual disorders, reduced or loss of financial income, social isolation, loss of sense of security, dependence on caregivers, etc [5-6]. Experiences of individuals with a chronic illness are highly subjective [7]. People with illness have different coping responses 
and varied coping resources, such as social support [8]. Social support has been studied extensively and refers to the degree of emotional and physical assistance perceived in one's life. Weiss proposed that individuals need a set of relationships over the course of their lives that can help organize their thinking and actions [9]. Fink, Graydon, and Ross reported that the presence of social support enhanced wellbeing by directly maximizing quality of life and by buffering the effects of adversity [10-11].

Older hemodialysis patients have physical and psychosocial stresses that negatively affect their quality of life. There is growing recognition of the association of self-care in chronically ill patients with improved quality of life [12]. Self-care is associated with several advantages, it improves coping with or adjustment to illness, increases sense of wellbeing, improves symptom control, decreases risk of complication, increases control and autonomy, increases functioning and finally enhances quality of life [13].

The purposes of this study were to measure self-care behaviors in elderly hemodialysis patients and to identify variables associated with self-care behaviors.

\section{Methods}

\subsection{Subjects and Data Collection}

The subjects were 113 elderly patients on HD who visited a tertiary hospital in Seoul between November $5^{\text {th }}$ and $30^{\text {th }}, 2012$, and consented to participate in the study. The questionnaire was administered to subjects who agreed to answer and participate. Data were collected by face-to-face interview using a structured questionnaire, which took 1015 minutes to complete. An Electronic Medical Record (EMR) was referred to regarding age, cause of ESRD, number of medications, comorbidities, serum phosphorus and potassium concentration, and mean weight gain between dialysis sessions.

\subsection{Instruments}

2.2.1. Self-Care Behaviors: The tool first invented by Song Mi-Ryeong (1999) and then modified by Cho Mi-Kyoung (2008), was used to measure the levels of self-care behaviors. It consists of 35 questions with a 5-point Likert scale. There are 7 subscales of medication ( 2 items), fistula management (6 items), management of physical problems (11 items), diet (6 items), exercise and rest (4 items), management of blood pressure and body weight ( 3 items), and social adjustment ( 3 items). The score for self-care behavior is distributed with 35-175 points and higher scores indicate a high performance of self-care.

2.2.2. Social Support: Levels of social support were measured using the tool first invented by Kim Ok-Su (1993) and then modified by Kim Gyeong-hee (1996). The scale consists of 18 items, with a 5-point Likert scale, generating the 2 subscales of Family and Medical staff. The score for social support is distributed with 18-90 points and a higher score reflected a higher level of perceived social support for that item.

2.2.3. Other Variables: Gender, age, marital status, education level, cause of ESRD, number of medications and comorbidities were determined from interviews and EMR reviews. Serum phosphorus and potassium concentration were measured in the clinical laboratory and mean weight gains between dialysis sessions were measured in the hemodialysis unit. 


\subsection{Data Analysis}

The SPSS WIN 15.0 program was used for analysis of collected data. Analysis included percentages, average, standard deviation, Pearson's correlation coefficients, and multiple regressions.

\subsection{Ethnical Consideration}

Standard ethical and legal points regarding the use of reporting subjects in research were as follows; salient, relative points were explained to all subjects. These guidelines included: participants' right to withdraw from the project, anonymity, limitations on the use of resulting data, use for research and or academic purposes only, and the possible destruction of sensitive materials.

\section{Results}

\subsection{General Characteristics of the Patients}

The general characteristics of the patients are shown in Table $1.61 \%$ of patients were male, with a mean age of 72.4 years. Diabetes and hypertension were the most common causes of ESRD. Subjects had comorbidities and were taking multiple medications.

Table 1. General Characteristics of Patents

\begin{tabular}{|l|l|l|}
\hline Variables & \multicolumn{2}{l}{$\mathrm{N}=113$} \\
\hline Gender & $\begin{array}{l}\text { Male } \\
\text { Female }\end{array}$ & $\begin{array}{l}\text { N(\%) or Mean } \pm \text { SD } \\
44(38.9)\end{array}$ \\
\hline Age(years) & & $72.4 \pm 6.8$ \\
\hline Marital status & $\begin{array}{l}\text { Single } \\
\text { Married }\end{array}$ & $\begin{array}{l}20(17.7) \\
93(82.3)\end{array}$ \\
\hline Years of education & Hiabetes & $11.0 \pm 4.4$ \\
\hline Cause of ESRD & Glomerulonephritis & $\begin{array}{l}60(53.1) \\
4(12.4)\end{array}$ \\
\hline Number of medication & & $10.2 \pm 3.8$ \\
\hline Comorbidities & & $2.1 \pm 1.1$ \\
\hline Serum phosphorus & & $4.9 \pm 1.0$ \\
\hline
\end{tabular}




\begin{tabular}{|l|l|l|}
\hline Potassium concentration & $4.6 \pm 0.6$ \\
\hline Inter-dialysis weight gain & & $2.2 \pm 0.8$ \\
\hline
\end{tabular}

ESRD, end stage renal disease

\subsection{Self-care Behaviors}

The mean score for self-care behaviors was 3.14 <Table 2>. Caring for an arteriovenous fistula, followed by taking medication showed the highest scores for selfcare activities, and engaging in social activities and diet control showed the lowest scores.

Table 2. Self-Care Behaviors

\begin{tabular}{|l|r|}
\hline Categories & $\mathrm{N}=113$ \\
\hline Medication & Mean \pm SD \\
\hline Fistula management & $3.86 \pm .7$ \\
\hline Management of physical problems & $3.91 \pm .3$ \\
\hline Diet & $3.41 \pm .4$ \\
\hline Exercise and rest & $2.71 \pm .5$ \\
\hline $\begin{array}{l}\text { Management of bloody pressure } \\
\text { and body weight }\end{array}$ & $3.14 \pm .7$ \\
\hline Social adjustment & $2.86 \pm .7$ \\
\hline Total & $2.10 \pm .5$ \\
\hline
\end{tabular}

\subsection{Social Support}

The mean score for social support was $3.14<$ Table 3>, and the social support showing the highest score was medical staff' support. 
Table 3. Social Support

\begin{tabular}{|c|c|}
\hline & $\mathrm{N}=113$ \\
\hline Categories & Mean $\pm S D$ \\
\hline Total & $76.8 \pm 10.11$ \\
\hline Family & $36.1 \pm 6.52$ \\
\hline Medical staff & $40.7 \pm 5.91$ \\
\hline
\end{tabular}

\subsection{Correlation between Self-care Behaviors and other Variables}

Significant negative correlations were observed for self-care behaviors and serum phosphorus $(\mathrm{r}=-.27, p<.001)$, and potassium concentration $(\mathrm{r}=-.35, p<.001)$. Significant positive correlation was observed between self-care behaviors and social support <Table $4>$.

\section{Table 4. Correlation between Self-care Behaviors and other Variables}

\begin{tabular}{|l|c|c|}
\hline & Self-care behaviors & $p$ \\
\hline Age(years) & -.284 & $<.001^{*}$ \\
\hline Years of education & -.231 & $.005^{*}$ \\
\hline Number of medication & .096 & .073 \\
\hline Comorbidities & -.121 & .081 \\
\hline Serum phosphorus & -.271 & $<.001^{*}$ \\
\hline Potassium concentration & & $<.001^{*}$ \\
\hline Inter-dialysis weight gain & -.352 & .062 \\
\hline Social support & -.089 & $<.001^{*}$ \\
\hline$p<.05$ & & \\
\hline
\end{tabular}




\subsection{Factors Affecting Self-care Behaviors in Elderly Hemodialysis Patients}

Age ( $p=.018)$, education level ( $p=.031)$, and social support $(p>.001)$, predicted value accounted for $36.8 \%$ of the variance in self-care behaviors in elderly hemodialysis patients $(\mathrm{F}=16.02, p>.001)<$ Table 5>.

\begin{tabular}{|c|c|c|c|c|}
\hline & Standardized Beta & $\mathrm{t}$ & $p$ & Adj $^{2}$ \\
\hline Constant & & & & \\
\hline & & & & \\
\hline Age & -0.21 & -2.47 & 0.018 & \\
\hline Educational level & 0.17 & 2.28 & 0.031 & \\
\hline Social support & 0.38 & 4.82 & $<.001$ & \\
\hline & & & & \\
\hline & & & & \\
\hline
\end{tabular}

\section{Discussion}

This study was conducted for measurement of self-care behaviors in elderly hemodialysis patients and to identify variables associated with self-care behaviors. We found that elderly patients on HD had low performance of self-care. Orem's self-care theory can be useful for healthcare providers in assisting patients in estimating their selfcare ability. Thus, healthcare providers should continue to gather information on self-care deficits in these individuals.

The self-care activities showing the highest scores were caring for an arteriovenous fistula, followed by taking medication, and engaging in social activities and diet control showed the lowest score. Patients with ESRD require continual care. Daily self-care includes managing a complex treatment regimen of dietary restrictions, fluid limitations, medications, and vascular access care. This day-to-day care is the responsibility of the patients. As renal function becomes significantly impaired, waste products begin to accumulate in the tissues, resulting in fatigue, neuropathy, irritability, and anorexia. Although anorexia is the body's way of limiting the waste products of digestion, it causes short-term weight loss and subsequent malnutrition, as evidenced by hypoalbuminemia. Baseline assessment of protein-energy nutritional status should include serum albumin level, anthropometry, subjective global assessment, degree of edema, and dietary interviews with further studies if the patient has an underlying disease characterized by proteinuria. A dietary plan should be presented to address caloric and protein intake and the need to restrict calcium, phosphorus, potassium, and sodium intake. These essential dietary changes should enhance nutrition, balance electrolytes, and promote the 
enjoyment of food. As kidney function declines, the elimination of phosphorus, an element found in nearly all foods, becomes less efficient, resulting in hyperphosphatemia. While the patient has residual kidney function, dietary restriction of phosphorus to less than $1 \mathrm{~g}$ per day is often sufficient to maintain phosphorus in the target range of 2.7 to 4.6 $\mathrm{mg} / \mathrm{dL}[14]$.

A significant relationship between self-care behaviors and physical factors was also observed. Several studies reported that post-self-care intervention, serum potassium levels were lower, showing improved levels [13]. In addition, mean weight gain measured between dialysis treatments was used as the measure of adherence to the regimen limiting fluid intake. Weight gains were calculated by subtracting the pre-dialysis weight of each participant from the post-dialysis weight at the last treatment. Weight gains were lower and therefore were improved for the self-care intervention. The patients in the behavioral contract with a family member or friend showed lower weight gains with statistical significance compared with the control group [13]. Further research should examine their contribution to outcomes in this patient population.

For chronically ill patients, illness can include a loss of social context, depleted support resources, and estrangement from a social network. Conley et al. reported that family members, health professionals, friends, and neighbors are major sources of support for dialysis patients [15]. The authors suggest that perceived social support improves the psychological wellbeing of the dialysis patient. Studies of in-center HD patients have reported that family support and a greater availability and involvement of the spouse were significantly associated with higher morale; family support and the availability of a confidant were associated with lower rates of illness exacerbation and social functioning difficulties [16]. The results of this study indicated that perceived social support was positively related to self-care ability. Nephrology professionals providing informational support to dialysis patients should include friends and partners, who can reinforce healthy behaviors and self-care. Health assessments should include an evaluation of the patient's support network to assess whether there is adequate support. Nephrology professionals can mobilize and enhance the support available to those undergoing HD through comprehensive assessment and appropriate intervention.

In conclusion, dialysis providers should recognize self-care behaviors as an important nursing issue for elderly hemodialysis patients. Self-care and self-efficacy training should also be provided to improve patients' confidence in performing self-care behaviors.

\section{References}

[1] A. J. Collins, B. Kasiske and C. Herzog, "Excerpts from the United States Renal Data System 2006 Annual Data Report", American Journal of Kidney Disease, vol. 49 (1 Suppl 1):A6-A7, (2007), pp. S1296.

[2] A. Kramer, V. Stel and C. Zoccali, "An update on renal replacement therapy in Europe: ERA-EDTA Registry data from 1997 to 2006", Nephorlogy Dialysis Transplantion, vol. 24, (2009), pp. 3557-3566.

[3] M. Moattari, M. Ebrahimi, N. Sharifi and J. Rouzbeh, "The effect of empowerment on the self-efficacy, quality of life and clinical and laboratory indicators of patients treated with hemodialysis: A randomized controlled trial", Health and quality of life outcomes, vol. 10, (2012), pp. 115.

[4] H. Qian and C. Yuan, "Factors associated with self-care self-efficacy among gastric and colorectal cancer patients", Cancer nursing, vol. 35, (2012), pp. E22-E31.

[5] Z. Moshtagh, K. Naeeni, M. Hamzezade and A. Arasteh, "The effects of nutrition education on mental health of the hemodialysis patients in maragheh, east-azerbaijan, iran”, Life Science Journal, vol. 10, (2013), pp. 382-386.

[6] M. Heidarzadeh, S. Atashpeikar and T. Jalilazar, "Relationship between quality of life and self-care ability in patients receiving hemodialysis", Iranian journal of nursing and midwifery research, vol. 15, (2010), pp. 66-71.

[7] G. Schussler, "Coping strategies and individual meanings of illness", Soc Sci Med., vol. 34, (1992), pp. 427-432.

[8] N. Woods, B. C. Yates and J. Primono, "Supporting families during chronic illness", Image Journal of Nursing Scholarship, vol. 21, (1989), pp. 46-50.

[9] R. S. Weiss, "The provisions of social relationships”, Englewood Cliffs, New Jersey, (1974). 
[10] S. V. Fink, "The influence of family resources and family demands on the strains and well-being of caregiving families", Nursing Research, vol. 44, (1995), pp. 139-146.

[11] J. E. Graydon and E. Ross, "Influence of symptoms, lung function, mood, and social support on level of functioning of patients with COPD", Research in Nursing \& Health, vol. 18, (1995), pp. 525-533.

[12] E. Seto, K. J. Leonard, J. A. Cafazzo, C. Masino, J. Barnsley and H. J. Ross, "Self-care and quality of life of heart failure patients at a multidisciplinary heart function clinic", Journal of Cardiovascular Nursing, vol. 26, (2011), pp. 377-385.

[13] C. Reid, J. Hall, J. Boys, S. Lewis and A. Chang, "Self-management of haemodialysis for End Stage Renal Disease: a systematic review”, The Joanna Briggs Library of systematic Reviews, vol. 9, (2011), pp. 69-103.

[14] K/DOQI, "clinical practice guidelines for bone metabolism and disease in chronic kidney disease", American Journal of Kidney Disease, vol. 42 (4 Suppl 3), (2003), pp. S1-201.

[15] J. A. Conley, H. J. Burton, A. K. DeNour and G. A. Wells, "Support systems for patients and spouses on home dialysis", International Journal of Family Psychiatry, vol. 2, (1981), pp. 45-54.

[16] M. Dimond, "Social support and adaptation to chronic illness: The case of maintenance hemodialysis", Research in Nursing \& Health, vol. 2, (1979), pp. 101-108.

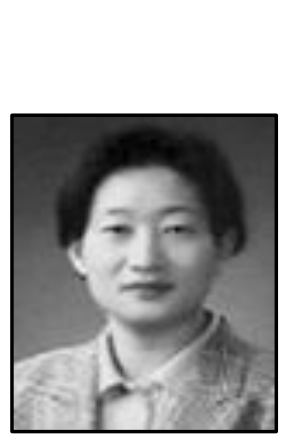

\begin{abstract}
Authors
Su-Jeong Han, she graduated onFeb. 2001: Ewha Womans Univ. PhD. Feb. 1997-Current: Konyang Univ. Professor. Research Interests: Medical-surgical nursing, chronic disease care. E-mail: sjhan@konyang.ac.kr
\end{abstract}

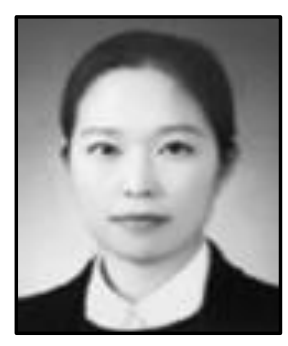

Hye-Won Kim, she graduated on Feb. 2012 : Seoul National Univ. PhD.Aug. 2014-Current : Seoul Women's College of Nursing, Professor. Research Interests: chronic kidney disease, Bio-behavioral factors E-mail: hwkim@snjc.ac.kr. Address: 38 GANHODAE-RO, SEODAEMUN-GU, SEOUL, KOREA, 120-742 